Electronic version of an article published as [International Journal of Production Economics, 2008, Vol. 111, No. 2, p. 697-706]

[DOI: http://dx.doi.org/10.1016/j.ijpe.2007.11.009] (c) [copyright Elsevier] 


\title{
DETERMINING THE MOST APPROPRIATE SET OF WEEKLY WORKING HOURS FOR PLANNING ANNUALISED WORKING TIME*
}

\author{
Amaia Lusa ${ }^{\dagger}$, Rafael Pastor, Albert Corominas \\ Research Institute IOC, School of Industrial Engineering of Barcelona, Universitat Politècnica de Catalunya, \\ Avda. Diagonal 647 p11, 08028 Barcelona (Spain) \\ \{amaia.lusa/rafael.pastor/albert.corominas\}@upc.edu
}

\begin{abstract}
Annualised hours — the irregular distribution of working hours over a year-allow companies to adapt capacity to demand, thus reducing overtime, the number of temporary workers and inventory costs. To avoid a significant deterioration in working conditions, many laws and agreements constrain the distribution of working time. One way of doing this is by specifying a finite set of weekly working hours and bounding the annual number of weeks of each type. Although this set has a great impact on the solution, it is usually agreed without taking all the available data (demand, costs, etc.) into consideration. This paper proposes a method for selecting the most appropriate set of weekly working hours and establishing an annual plan or working time for each worker as a way of optimising service level. To this end, two mathematical programming models are proposed. By means of a computational experiment, it is shown that one of the models can be solved in short computing times and can thus be used as a decision-making tool.
\end{abstract}

Keywords: human resources, manpower planning, annualised hours, aggregated planning

\footnotetext{
* ACKNOWLEDGMENTS: Paper supported by Project DPI2004-05797.

${ }^{\dagger}$ CORRESPONDING AUTHOR:

Amaia Lusa

Research Institute IOC, School of Industrial Engineering of Barcelona

Universitat Politècnica de Catalunya

Avda. Diagonal, 647, planta 11

08028 Barcelona (Spain)

Tel. +34934011705

Fax +34 934016605

e-mail: amaia.lusa@upc.edu
} 


\section{INTRODUCTION}

Annualising working hours $(\mathrm{AH})$ consists in hiring workers for a certain number of hours per year and distributing these hours irregularly over the year in order to accommodate fluctuations in demand. This makes better use of potential capacity because it can be adapted more closely to demand over time. This flexibility in the use of human resources is especially useful in service organisations and in manufacturing organisations where products cannot be inventoried (e.g. are perishable). However, as is discussed below, AH often means worse working conditions for staff and the need to solve complicated working time planning problems.

At the outset, the implications for workers on annualising hours may include a significant drop in the quality of their personal and leisure time. Hence, annualised working-hour systems must be negotiated between companies and workers. This usually involves some kind of compensation (e.g. shorter working hours, pay rises) being offered to workers in exchange for greater flexibility. Furthermore, an appropriate set of conditions must be devised for distributing working time in order to avoid—or at least reduce—-the system's drawbacks. There are innumerable ways of defining these conditions, since they may be based on legal requirements or result from collective bargaining. This is one of the reasons for the wide range of problems that arise in planning annualised hours.

These problems have hitherto been given little attention in the literature. There are papers that deal with production and working time planning problems in which some kind of flexibility is considered (e.g. Wild and Schneeweiss, 1993; Lagodimos and Mihiotis, 2006). However, annualised hours is a subject that has remained largely unexplored. In fact, Hung (1999a), Hung (1999b), Grabot and Letouzey (2000) and Azmat and Widmer (2004) all draw attention to the fact that the concept of annualised hours is surprisingly absent from the literature on planning and scheduling. Some authors deal with different versions of the working time planning problem (e.g. Hung, 1999a; Hung, 1999b; Vila and Astorino, 2001; Corominas et al., 2002; Azmat and Widmer, 2004; Azmat et al., 2004), but most papers (e.g. Lynch, 1995; MacMeeking, 1995 and Mazur, 1995) only discuss AH from a qualitative point of view. 
Vila and Astorino (2001) give an example of a real AH scenario: an agricultural machinery manufacturer that introduced AH was able to reduce overtime and the use of temporary workers by $94 \%$ and $53 \%$, respectively. Due to the great variety of production systems, AH gives rise to a considerable number of problems. In Corominas et al. (2004) the characteristics of the planning problem are discussed and a classification scheme is proposed, resulting in thousands of different cases. We have already solved some problems of this type (see Corominas et al., 2002 and Corominas et al., 2007) and obtained solutions to show that when an AH system is compared to a traditional system (regular working hours), the costs due to overtime, temporary workers, outsourcing, drops in demand and, in some cases, inventory, are diminished. In all cases, in spite of the large size of the models, Mixed Integer Linear Programming (MILP) has proved to be an efficient tool for solving these problems.

The conditions devised to overcome the drawbacks that annualisation implies for workers could be designed to avoid excessive temporary concentrations of the work load (for instance, an upper bound on the number of weeks with a number of working hours greater than a certain predefined value) or to ensure that the organisation of working time includes positive features, such as a minimum number of weeks with shorter working hours (Corominas et al., 2002). One way of doing this is to use a finite set of weekly working hours and to bound the number of times that each element in the set is assigned to each worker over the year. This is a means of implicitly incorporating many important conditions, thereby simplifying the process of establishing the annual working time plan. It also makes the plan more comprehensible and easier to explain. Actually, this idea has been applied in French companies to deal with annualised hour systems, which have been allowed and fostered by the so-called 35-hour working week law (MES, 2005).

As could be expected, the characteristics of the finite set of weekly working hours may considerably influence the quality of the optimal planning (i.e. how well capacity can be adjusted to demand). This set may result from negotiations between employees and the company, without an accurate assessment of the consequences of the scheme on the cost or on the level of quality of service. In such cases, the set of weekly working hours must be taken as a datum for planning. In this paper, this assumption is relaxed in order to design a procedure which allows not only planning working hours based on a pre-determined set but also determining the most appropriate set of weekly working hours (i.e. the one that best allows capacity to be adjusted to demand). 
The remainder of this paper is structured as follows: Section 2 presents the problem; Section 3 contains a brief discussion of the objective function; Section 4 describes the planning models; Section 5 reports on the computational experiment; and Section 6 contains the conclusions.

\section{DESCRIPTION OF THE PROBLEM}

The problem consists in determining the number of weekly working hours for each member of staff at a service centre and for each normal working week in the planning horizon (a year, for instance), with the objective of optimising a utility function.

In some countries, collective bargaining agreements do not permit overtime and tasks are sometimes too difficult to be performed by temporary workers. For the purposes of this paper, as overtime and temporary workers are not considered, a capacity shortage is allowed for in certain weeks. Based on these assumptions, staff costs are the same in all feasible situations. Thus, capacity must be distributed in order to optimise the service level. Section 3 contains a discussion of the most appropriate objective function.

It is assumed that the number of working hours must belong to a finite set, which must also be determined according to the utility function and to other additional rules agreed on by managers and workers. Moreover, the number of annual working weeks of each type of week can be previously established or bounded.

From the outset, the following two approaches for determining the most appropriate set of working weeks had to be considered:

1. The number of hours corresponding to each type of working week must be chosen from a finite list.

2. The number of hours corresponding to each type of working week can take any value belonging to an interval defined by a lower and upper bound. 
To ensure a fair outcome, in both cases it is assumed that the number of weeks in which each type of working week is assigned has to be the same for all workers.

Obviously, the set of feasible solutions corresponding to the first approach is, strictly speaking, a subset of that belonging to the second one. However, the latter may possibly give rise to solutions that are difficult to implement, since it assumes that all fractional values are admissible for the weekly working time. Whatever the case, the two approaches could be adopted to obtain workable solutions, provided that the corresponding models were solvable in a reasonable amount of time.

Additional conditions to be met by the solution may stem from legal requirements or from collective bargaining agreements between the management and workers. As it is impossible to establish an exhaustive list of a priori conditions, in this paper the most commonly adopted conditions are considered in order to build a basic model for the problem. To adapt the model to specific cases, constraints should be added or removed.

To avoid overburdening workers over a number of consecutive high-demand periods, an additional constraint, which is provided for in France’s 35-hour working week law, is considered: the average number of weekly working hours for any set of twelve consecutive working weeks is upper bounded.

The characteristics of the problem are summarised below:

- The main condition of $\mathrm{AH}$, which is that the number of annual working hours is upper bounded, must be fulfilled.

- The number of weekly working hours must belong to a finite set, which must be pre-determined. Two possibilities are considered:

1. Each type of working week must be chosen from a list of available working weeks. For example, three types of working weeks must be chosen from nine available ones: the first type is to be chosen from $\{36$, $37,38\}$, the second from $\{40,41,42\}$ and the third from $\{43,44$ and 45$\}$. 
2. The number of hours corresponding to each type of working week is lower and upper bounded. For example, the lower and upper bounds corresponding to the three types of working weeks could be [35, 38], [40, 43] and [43.5, 48], respectively.

- The number of times in a year in which each type of working week is assigned must be the same for all workers and is lower and upper bounded.

- The average number of working hours for a group of $L$ consecutive normal working weeks cannot be greater than $h_{L}$ hours/week.

- Each worker has a certain number of holiday weeks that are previously agreed on (for example, two consecutive holiday weeks in winter and four consecutive holiday weeks in summer).

- Overtime is not permitted.

- Hiring temporary workers is not possible.

- A utility function, which is discussed in following section, must be optimised.

\section{DISCUSSION OF THE OBJECTIVE FUNCTION}

As the cost is the same for all feasible solutions (there is no overtime or temporary workers), a utility function related to service level must be considered.

It is assumed that all customers will be served when they go to the service centre. Thus, demand must be met at all times, but if there is a capacity shortage the service level will not be as good as is desirable (workers may spend too little time serving customers). If the required capacity (which is fixed according to foreseen demand and the optimal service level, as in a queue system) is greater than the actual capacity, the service level inevitably worsens. Of course, to make the assumption that demand will always be covered would be unsustainable if relative capacity shortages (i.e. capacity shortage divided by the required capacity) were high.

High relative shortages must be avoided; if a capacity shortage represented a small proportion of the required capacity—although less attention would be paid to some customers-workers could meet the demand by making a little extra effort. The maximum relative capacity shortage, $D$, a function that is relatively simple to 
minimise, is a suitable objective function. The service level during the worst period is optimised and high capacity shortages are avoided.

The main inconvenience of this objective function $([\mathrm{MIN}] \mathrm{z}=D)$ is that once the maximum relative capacity shortage has been minimised, it makes no difference whether there is a capacity shortage in other weeks, provided that relative capacity shortages are not higher than the minimum (Figure 1 shows the required capacity, the obtained capacity and the shortage obtained for every week by solving an example using Model M1—described below—using this objective function). If $D$ is maintained at its optimal value, it is obviously preferable to have the smallest amount of shortages in all the weeks included in the planning horizon.

\section{[INSERT FIGURE 1]}

Figure 1: Minimise maximum relative capacity shortage.

In order to obtain low relative capacity shortages for every week, it is possible to break the ties between optimal solutions by considering a secondary objective function, which is weighted and then added to the first one: namely, the sum of weekly relative capacity shortages. In accordance with this, the objective function that must be minimised is the weighted sum of the following two terms: (i) the maximum relative capacity shortage; and, (ii) the sum of the weekly relative capacity shortages. Similarly to Figure 1, Figure 2 shows the required capacity, the obtained capacity and shortage profiles for every week. In this case, the results were obtained by means of Model M1 using the aforementioned objective function and the same data as in Figure 1. Thus, as can be seen, the capacity obtained adapts better to that required and the service level improves, since the sum of the shortages is less than that corresponding to the mere minimisation of $D$.

\section{[INSERT FIGURE 2]}

Figure 2: Minimise weighted sum of maximum relative capacity shortage and the sum of weekly relative capacity shortages.

Achieving the same solution by means of regular hours and overtime instead of annualising hours would be very expensive and, in some cases, legally unfeasible. Figure 3 shows the overtime that would be necessary 
to match the shortages obtained in the solution shown in Figure 2 (as it can be observed in Figure 3 there is a significant fluctuation in regular hours, which is due to the distribution of workers' holidays). An annual total of 124.3 hours of overtime per worker would be required, which far exceeds the amount permitted by law in many countries (in Spain, for example, the maximum annual overtime allowed by law is 80 hours per worker). This illustrates that the flexibility of AH is economically advantageous.

\section{[INSERT FIGURE 3]}

Figure 3: Required capacity, obtained capacity (standard working hours plus overtime) and shortage profiles obtained when regular hours and overtime are used instead of AH and capacity shortages match those obtained with AH (see Figure 2).

\section{MODELS}

\subsection{Model M1}

The first model corresponds to the approach in which each type of working week must be chosen from an available set.

We will use the following notation.

\section{Data}

W Set of staff members.

$N \quad$ Number of staff members $(=|W|)$.

$T \quad$ Weeks in the planning horizon (usually 52).

$S_{i} \quad$ Set of available weeks per worker $i$ (weeks in the planning horizon, minus the worker's holiday weeks), $\forall i \in W$.

H Upper bound for annual working hours.

K Number of types of working weeks to be determined.

$J_{k} \quad$ Type k working week must be chosen from the set of types of working weeks $J_{k}(\mathrm{k}=1, \ldots, K)$.

JT Set of types of working weeks $\left(J T=\cup_{k=1}^{K} J_{k}\right)$. 
$j_{w} \quad w$ is one of the types of working weeks from which type $j_{w}\left(j_{w} \in[1 . . K]\right)$ working week must be chosen $(\forall w \in J T)$.

$n h_{w} \quad$ Number of hours corresponding to type $w(\forall w \in J T)$ working week.

$l b_{k}, u b_{k} \quad$ Lower bound and upper bound for the number of type $k$ working weeks that must be performed by each worker $(k=1, \ldots, K)$.

$L, h_{L} \quad$ The average number of working hours, in a group of $L$ consecutive weeks, cannot be greater than $h_{L}$ (for example, $L=12$ and $h_{L}=44$ hours).

$e_{t} \quad$ Working hours required during week $t(\mathrm{t}=1, \ldots, T)$, considering the time that a worker would need to complete the task, and fixed according to the foreseen demand of the task and the desired service level.

$\alpha, \beta \quad$ Weight given to the two criteria considered in the objective function (maximum relative shortage and sum of relative shortages).

Variables

Binary variable that indicates whether worker $i$ performs a type $w$ working week during week $t$ $\left(\forall i \in W ; \forall t \in S_{i} ; \forall w \in J T\right)$.

$y_{w}$

Binary variable that indicates whether type $w$ working week has been chosen $(\forall w \in J T)$.

$a_{k}$ Integer variable that indicates the number of weeks in which the selected type $k$ working week is assigned to workers $(\mathrm{k}=1, \ldots, K)$. In fact, this variable can be said to be a non-negative real variable because it can only take integer values (it is expressed in constraint (5) as the sum of binary variables).

$d_{t}^{-} \quad$ Non-negative real variable that indicates the capacity shortage; that is to say, the anticipated required capacity that cannot be met by the staff in week $t(\mathrm{t}=1, \ldots, T)$.

$D \quad$ Non-negative real variable that indicates the maximum relative shortage, relative shortages being the shortages divided by their corresponding requirements. 


$$
\begin{aligned}
& {[M I N] Z=\alpha \cdot D+\beta \cdot \sum_{t=1}^{T} \frac{d_{t}^{-}}{e_{t}}} \\
& D \geq \frac{d_{t}^{-}}{e_{t}} \\
& \sum_{\forall t \in S_{i}} \sum_{\forall w \in J T} n h_{w} \cdot X_{i w t} \leq H \\
& \sum_{\forall w \in J T} x_{i w t}=1 \\
& \sum_{\forall t \in S_{i}} \sum_{\forall w \in J_{k}} x_{i w t}=a_{k} \\
& l b_{k} \leq a_{k} \leq u b_{k} \\
& \sum_{\forall w \in J_{k}} y_{w}=1 \\
& \sum_{\forall i \in W} \sum_{\forall t \in S_{i}} x_{i w t} \geq y_{w} \\
& \sum_{\forall i \in W} \sum_{\forall t \in S_{i}} x_{i w t} \leq N \cdot u b_{j_{w}} \cdot y_{w} \\
& \sum_{(\forall i \in W) \mid\left(t \in S_{i}\right)} \sum_{\forall w \in J T} h_{w} \cdot x_{i w t}+d_{t}^{-} \geq e_{t} \\
& \sum_{t=j-L+1}^{j} \sum_{\forall w \in J T} h_{w} \cdot x_{i w t} \leq h_{L} \cdot L \quad \forall i \in W ; j=L, \ldots, T \mid[j-L+1, \ldots, j] \in S_{i}
\end{aligned}
$$

Finally, variables $x_{i w t}$ and $y_{w}$ are binary, whilst variables $a_{k}, d_{t}^{-}$and $D$ are real and non-negative.

The objective function to minimise (1) is the weighted sum of (i) the maximum relative capacity shortage, and (ii) the sum of weekly relative capacity shortages, where $\alpha, \beta>0$; (2) expresses that $D$ is the maximum relative capacity shortage; (3) sets the upper bound for the number of annual working hours for each worker; (4) requires that one type of working week be assigned to each worker and each available week; (5) and (6) set the lower and upper bound for the number of times that each type of working week is assigned, which must the same for all workers; (7) requires that one type of working week be chosen from each available set; 
Equations (8) and (9) imply that the variable $y_{w}$ takes value 1 if working week of type $w$ is assigned and 0 otherwise; (10) is the weekly balance between the number of required working hours, the number of working hours performed by the staff and the capacity shortage; and, finally, (11) sets an upper bound on the average weekly working hours for any subset of $L$ consecutive normal working weeks.

\subsection{Model M2}

This second model corresponds to the approach in which the number of hours corresponding to each type of working week can take any real value belonging to an interval defined by a lower and an upper bound. An additional notation is defined.

Data

$h m_{k}, h M_{k} \quad$ Lower bound and upper bound for the hours in type $k(k=1, \ldots, K)$ working week.

\section{Variables}

$h_{k} \quad$ Non-negative real variable that indicates the number of working hours corresponding to type $k$ $(\mathrm{k}=1, \ldots, K)$ working week.

$$
\begin{aligned}
& {[M I N] Z=\alpha \cdot D+\beta \cdot \sum_{t=1}^{T} \frac{d_{t}^{-}}{e_{t}}} \\
& D \geq \frac{d_{t}^{-}}{e_{t}} \\
& \sum_{k=1}^{K} h_{k} \cdot\left(\sum_{\forall t \in S_{i}} X_{i k t}\right) \leq H \\
& \sum_{k=1}^{K} X_{i k t}=1 \\
& \sum_{\forall t \in S_{i}} X_{i k t}=a_{k}
\end{aligned}
$$


$l b_{k} \leq a_{k} \leq u b_{k}$

$\sum_{k=1}^{K} h_{k} \cdot\left(\sum_{(\forall i \in W) \mid\left(t \in S_{i}\right)} X_{i k t}\right)+d_{t}^{-} \geq e_{t}$

$\sum_{k=1}^{K} h_{k} \cdot\left(\sum_{t=j-L+1}^{j} x_{i k t}\right) \leq h_{L} \cdot L$

$h m_{k} \leq h_{k} \leq h M_{k}$

$$
\begin{array}{r}
k=1, \ldots, K \\
t=1, \ldots, T \\
\forall i \in W ; j=L, \ldots, T \mid[j-L+1, \ldots, j] \in S_{i} \\
k=1, \ldots, K
\end{array}
$$

Finally, variables $x_{i k t}$ are binary, whilst variables $a_{k}, h_{k}, d_{t}^{-}$and $D$ are real and non-negative.

The objective function and certain constraints are the same as in M1. Equation (12) sets the upper bound for the number of annual working hours for each worker; (13) is the weekly balance between the number of required working hours, the number of working hours performed by the staff and the capacity shortage; (14) sets an upper bound on the average weekly working hours for any subset of $L$ consecutive normal working weeks; and, finally, (15) sets the lower and the upper bound for the number of working hours for each type of working week.

Equations (12), (13) and (14) include the sum of the values of the variables $h_{k}$ by a sum of binary variables $x_{i k k}$. In general, it is possible to linearise the value of a real variable, $R$, by a sum of binary variables (which is an integer variable, $I$ ) as follows:

- $R \cdot I$ is replaced by a new real variable $S$;

- $I$ is expressed as $\sum_{n=n \min }^{n \max } n \cdot b_{n}, b_{n}$ being binary variables and $n$ min and $n$ max the lower and upper bound for integer variable $I$;

- Constraint $\sum_{n=n \min }^{n \max } b_{n}=1$ is added;

- Finally, the following equations are added:

$$
\begin{array}{ll}
S-n \cdot R \leq M \cdot\left(1-b_{n}\right) & n=n \min , \ldots, n \max \\
n \cdot R-S \leq M \cdot\left(1-b_{n}\right) & n=n \min , \ldots, n \max
\end{array}
$$


This technique was used to linearise Equations (12), (13) and (14), thus giving rise to a MILP model (which will simply be called Model M2 hereinafter). Unfortunately, linearisation entails a substantial increase in the number of constraints in the model, which finally turned out to be inefficient and Model $M 1$ was clearly much more effective (see Section 5). Taking this into account, we believe that there is little point in giving any further detailed description of the linearisation of $M 2$.

\section{COMPUTATIONAL EXPERIMENT}

A computational experiment was performed in order to evaluate the effectiveness of the models. Whilst Model M1 could be solved very efficiently, the linear version of Model M2 turned out to be hard to solve and in most instances it was even impossible to obtain any feasible solutions in a reasonable time.

The data set used for the computational study was designed to cover the great majority of real cases specific to the problem. It allowed us to test the effectiveness of the models and their sensitivity in relation to the parameters that define an instance. The values used were as follows:

- $|W|=$ staff sizes of $10,20,30,40$ and 50 workers.

- $T=52$ weeks;

- Each worker has two weeks of uninterrupted holidays in winter and four in summer. The temporary holiday period for each worker was randomly fixed. This way of generating workers' holidays is by no means supposed to be realistic and does not have any advantages from the point of view of resolving the problem. Furthermore, it ensures variety in the reduction of staff availability as a result of holidays.

- Three different patterns of required capacity over the year were considered (see Figure 4): (1) nonseasonal capacity pattern (AH may also be useful under such circumstances as not all workers take their holidays at the same time; under a system of regular hours, the capacity would not be constant and the service level would not be optimised); (2) seasonal pattern with one peak; and (3) seasonal pattern with two peaks. In each case, the total required capacity was equal to the total available capacity and a random noise was added. 
- Three types of working weeks were considered - the number used in a real case known to the authors, which is small but provides sufficient flexibility. Moreover, the smallness of the finite set of types of working weeks is actually one of the main characteristics of this particular modality of AH and one of the main reasons for choosing it. However, a larger number of working weeks (maybe four or five) could be considered without making a significant impact on the size of the model.

- In the case of Model M1, three types of working weeks had to be chosen from the following 23 ones that were available: $\{28,29,30,31,32,33,34,35\},\{36,37,38,39,40,41,42,43\}$ and $\{44,45,46,47,48$, $49,50\}$.

- In the case of Model M2, the lower and upper bound for the hours corresponding to each of the three types of working week were [28-35], [35.1-43], and [43.1-50] hours. These intervals correspond to the lists considered for Model M1. The minimum and maximum number of working hours established for both models are reasonable (most countries' laws allow these values). The first interval (list) was a "light” working week, the second a "normal” working week and the third a "heavy" working week, which may help in negotiations on the bounds laid down with regard to the number of times per year that each type of working week has to be assigned.

- Each of the three types of working week has to be assigned in a number of weeks between 6 and 34 .

- $\alpha=0.99$ and $\beta=0.01 / T$ ( $\beta$ is divided by $T$ to standardise the second part, whose upper bound is equal to $T)$.

\section{[INSERT FIGURE 4]}

Figure 4: Required capacity patterns

For each combination of $W$ and pattern of required capacity, 20 instances were generated, resulting in a total of 300 instances for each model. The experiment was performed using an ILOG CPLEX 9.0 and a Pentium IV PC at $3.0 \mathrm{GHz}$ with $512 \mathrm{Mb}$ of RAM. Absolute and relative MIP gap tolerances were set to 0.001 and the maximum computing time was set to 3,600 seconds.

The size of the models is given in Table 1 . In Table 2, one can see the minimum $\left(t_{\min }\right)$, the average $(\bar{t})$ and the maximum $\left(t_{\max }\right)$ computing times for each size of model. \%Opt expresses the percentage of instances that 
were solved to optimality; \%5Opt and $\%>5 O p t$ are the percentages of instances whose difference from the best bound is lower and greater than 5\%, respectively, when the maximum computing time of 3,600 seconds was achieved; finally, \%NonFeas shows the percentage of instances without a feasible solution after 3,600 seconds.

Solving Model M1 always gives a feasible solution and an optimal or near optimal solution in most instances. Though the efficiency of the model decreases with the number of workers, note that, as is stated in Azmat and Widmer (2004), companies with over 20 workers are mainly made up of small teams of workers specialised in performing certain types of tasks, so the planning problem can be divided into a set of subproblems. Since the working time of workers belonging to different sections or teams can be planned and programmed separately, it can be concluded that Model $M 1$ can be solved very efficiently for most real situations (note that computing times are short, considering the problem that is being solved).

Though the maximum computing time is used up in all instances, solving Model M2 provides solutions that are of worse quality than those obtained with Model $M 1$ (the value of the objective function of the solution given by Model M1 is always better—67\%, on average—-than that obtained by solving Model M2).

\section{[INSERT TABLE 1]}

Table 1: Size of the models.

\section{[INSERT TABLE 2]}

Table 2: Computing times (in seconds).

Computing times increase as the number of staff workers increases, which determines the size of the models. In Figure 5, the influence of the pattern of required capacity on computing times for Model M1 can be observed. Computing times for the second and third patterns of required capacity (seasonal patterns) are generally longer than for the first pattern. Also, the percentage of solutions that are proven to be optimal within the maximum computing time of 3,600 seconds (100\%, $85.33 \%$ and $86.49 \%$ for the first, second and third pattern of required capacity, respectively) is slightly smaller for the seasonal patterns. This is not 
surprising given that in the case of a non-seasonal requirement it is easier to accommodate capacity to the patterns.

\section{[INSERT FIGURE 5]}

Figure 5: Influence of pattern of required capacity on computing times.

Finally, the maximum relative capacity shortages and average relative capacity shortages obtained with model M1 (by number of workers and pattern of required capacity in Table 3) show that with a small finite set of types of working weeks it is possible to adapt capacity to demand in a very efficient way. The values on Table 3 also show that, as it is reasonable, big teams provide more flexibility than small teams (solutions improve as the number of workers increase), even though with 10 workers solutions are also good. These results also strengthen the idea of having a medium or big team of cross-trained workers rather than a small team of specialized workers (Corominas et al., 2006).

\section{[INSERT TABLE 3]}

Table 3: Maximum relative capacity shortages and average relative capacity shortages (model M1).

\section{CONCLUSIONS}

Annualising working hours is an efficient, economical way of adjusting capacity to that required for seasonal demand and obtaining flexibility in the distribution of annual working hours. This paper presents a specific problem of planning working hours in services in which the number of weekly working hours must belong to a finite set, and in which overtime and temporary workers are not permitted. Usually, the finite set of working weeks is negotiated between the company and workers without taking into account that its influence on the final solution is highly significant. This paper presents two MILP models for solving the planning problem and also for determining the most appropriate set of weekly working hours, by optimising a function related to the service level. 
A computational experiment showed that Model M1 (which considers lists of available working weeks from which the set that will actually be used must be chosen) is an efficient tool for reaching an optimal solution to the problem, even in the case of large instances. Model M2 (which determines each type of working week by means of a real variable) entails a substantial number of constraints and takes a long time to solve.

The proposed planning procedure can be a useful tool for negotiating the best set of types of working weeks. This set complies with the conditions that workers and a company may agree on and optimises an objective function related to the service level.

Further research could explore other versions of the problem, by, for example, allowing a type of working week to be assigned a number of times that is not the same for all workers. Such a system might have the disadvantage of not treating all workers fairly, but it allows for a higher level of flexibility and therefore could adjust capacity even better to requirement fluctuations. Other assumptions that could be relaxed in future research are the fact that holidays are agreed on in advance (even though this is the most common case, in some situations holidays could be considered as variables to be determined by the model) and that all workers are considered equal (different tasks, and different categories of workers who perform these tasks to varying degrees of efficiency could also be considered).

\section{REFERENCES}

Azmat, C., Widmer, M., 2004. A case study of single shift planning and scheduling under annualized hours: A simple three step approach. European Journal of Operational Research 153, 148-175.

Azmat, C., Hürlimann, T., Widmer, M., 2004. Mixed integer programming to schedule a single-shift workforce under annualized hours. Annals of Operations Research 128, 199-215.

Corominas, A., Lusa, A., Pastor, R., 2002. Using MILP to plan annualised working hours. Journal of the Operational Research Society 53, 1101-1108.

Corominas, A., Lusa, A., Pastor, R., 2004. Characteristics and classification of the annualised working hours planning problems. International Journal of Services, Technology and Management 5, 435-447.

Corominas, A., Lusa, A., Pastor, R., 2007. Using a MILP model to establish a framework for an annualised hours agreement. European Journal of Operational Research 177, 1495-1506. 
Corominas, A., Pastor, R., Rodríguez, E., 2006. Rotational allocation of tasks to multifunctional workers in a service industry. International Journal of Production Economics 103, 3-9.

Grabot, B., Letouzey, A., 2000. Short-term manpower management in manufacturing systems: new requirements and DSS prototyping. Computer in Industry 3, 11-29.

Hung, R., 1999a. A multiple-shift workforce scheduling model under annualized hours. Naval Research Logistic 46, 726-736.

Hung, R., 1999b. Scheduling a workforce under annualized hours. International Journal of Production Research 37, 2419-2427.

Lagodimos, A. G., Mihiotis, A. N., 2006. Overtime vs. regular shift planning decisions in packing shops. International Journal of Production Economics 101, 246-258.

Lynch P., 1995. Annual Hours: An idea whose time has come. Personnel Management November, 46-50.

MacMeeking J., 1995. Why Tesco’s new composite distribution needed annual hours. International Journal Retail Distribution Management 23, 36-38.

Mazur L., 1995. Coming: the annual workweeks. Across the Board 32, 42-45.

MES: Ministère de l'Emploi et de la Solidarité: www.35h.travail.gouv.fr 28/02/2005. France.

Vila, G.F.E., Astorino, J. M., 2001. Annualized hours as a capacity planning tool in make-to-order or assemble-to-order environment: an agricultural implements company case. Production Planning \& Control 12, 388-398.

Wild, B., Schneeweiss, C., 1993. Manpower capacity planning - A hierarchical approach. International Journal of Production Economics 30-31, 95-106. 


\section{LIST OF FIGURES}

Figure 1: Minimise maximum relative capacity shortage.

Figure 2: Minimise the weighted sum of maximum relative capacity shortage and the sum of weekly relative capacity shortages.

Figure 3: Required capacity, obtained capacity (standard working hours plus overtime) and shortage profiles obtained when regular hours and overtime are used instead of AH and capacity shortages match those obtained with AH (see Figure 2).

Figure 4: Required capacity patterns

Figure 5: Influence of pattern of required capacity on computing times.

\section{LIST OF TABLES}

Table 1: Size of the models.

Table 2: Computing times (in seconds).

Table 3: Maximum relative capacity shortages and average relative capacity shortages (model M1). 


\section{FIGURE 1}

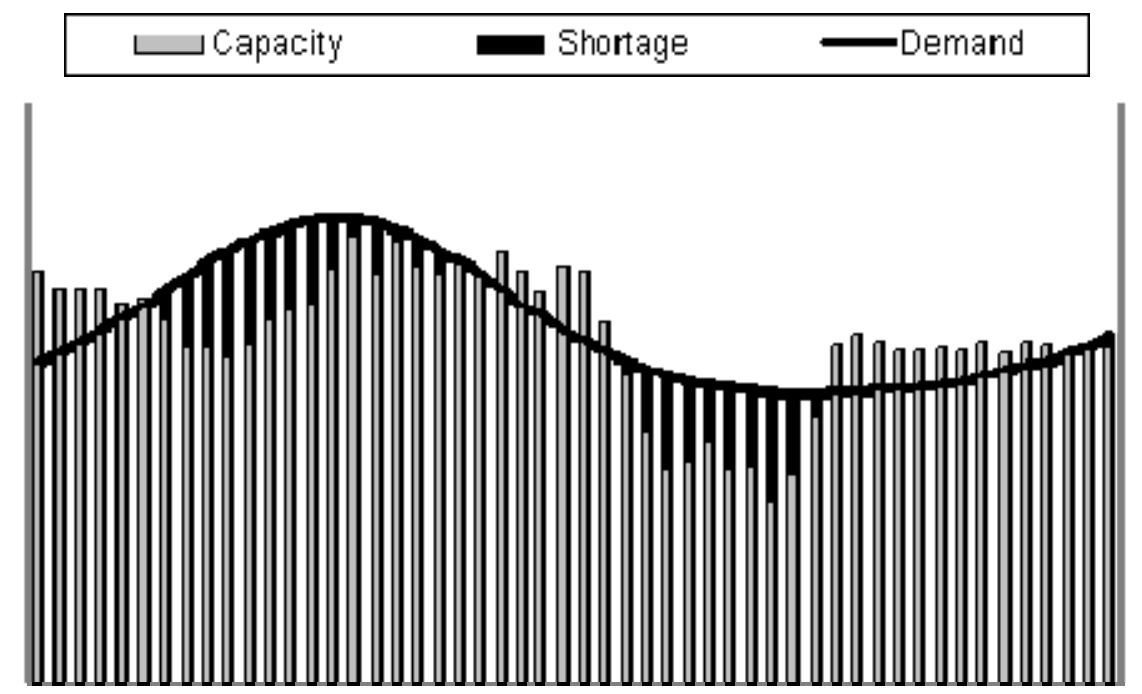

Figure 1: Minimise maximum relative capacity shortage. 
Figure 1: Minimise maximum relative capacity shortage. 


\section{FIGURE 2}

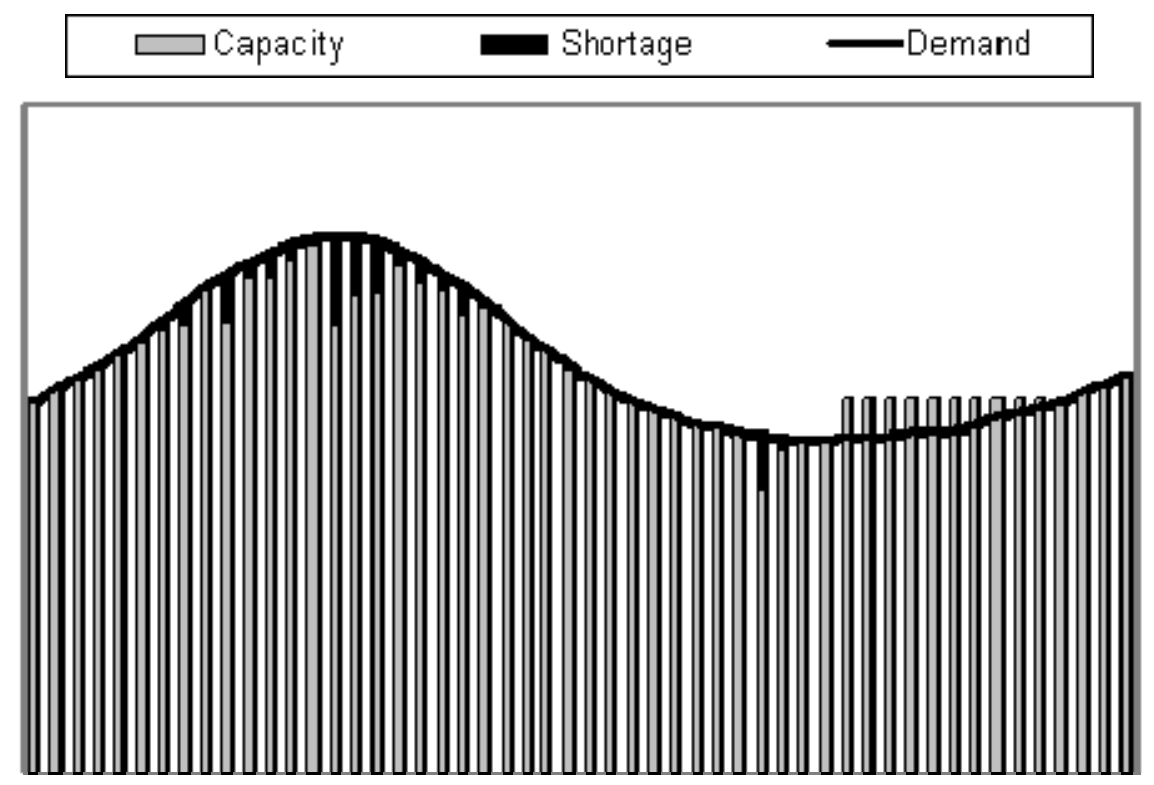

Figure 2: Minimise the weighted sum of maximum relative capacity shortage and the sum of weekly relative capacity shortages. 
Figure 2: Minimise the weighted sum of maximum relative capacity shortage and the sum of weekly relative capacity shortages. 


\section{FIGURE 3}

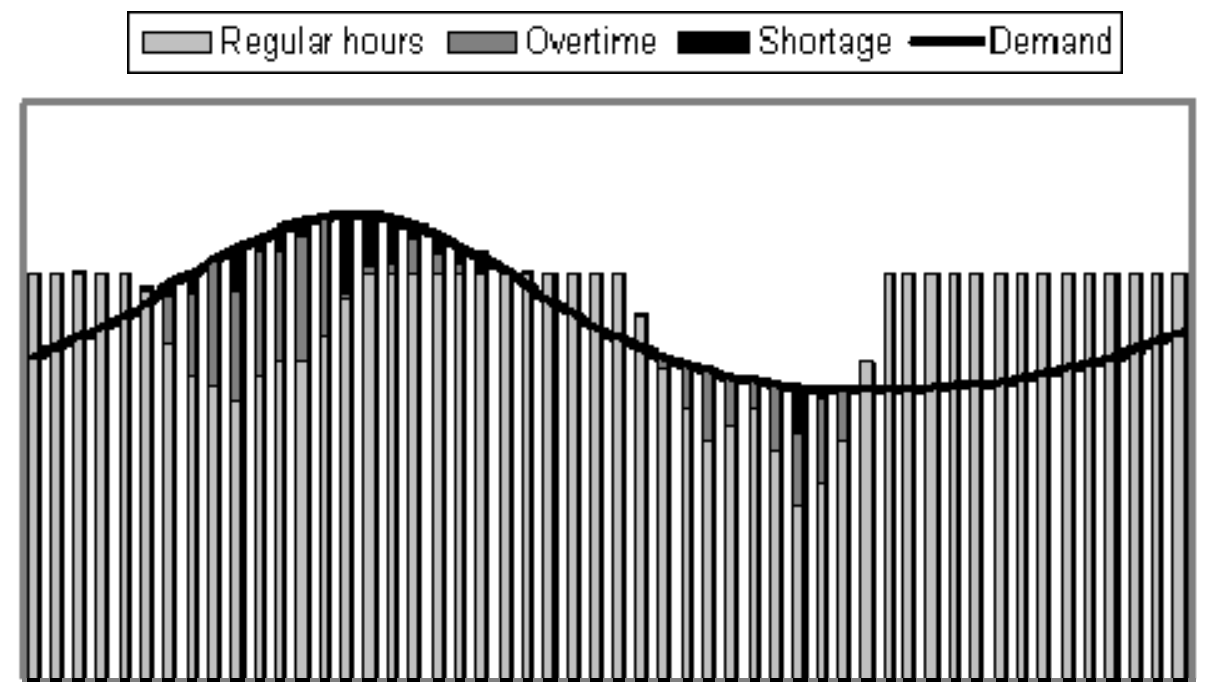

Figure 3: Required capacity, obtained capacity (standard working hours plus overtime) and shortage profiles obtained when regular hours and overtime are used instead of AH and capacity shortages match those obtained with AH (see Figure 2). 
Figure 3: Required capacity, obtained capacity (standard working hours plus overtime) and shortage profiles obtained when regular hours and overtime are used instead of AH and capacity shortages match those obtained with AH (see Figure 2). 


\section{FIGURE 4}

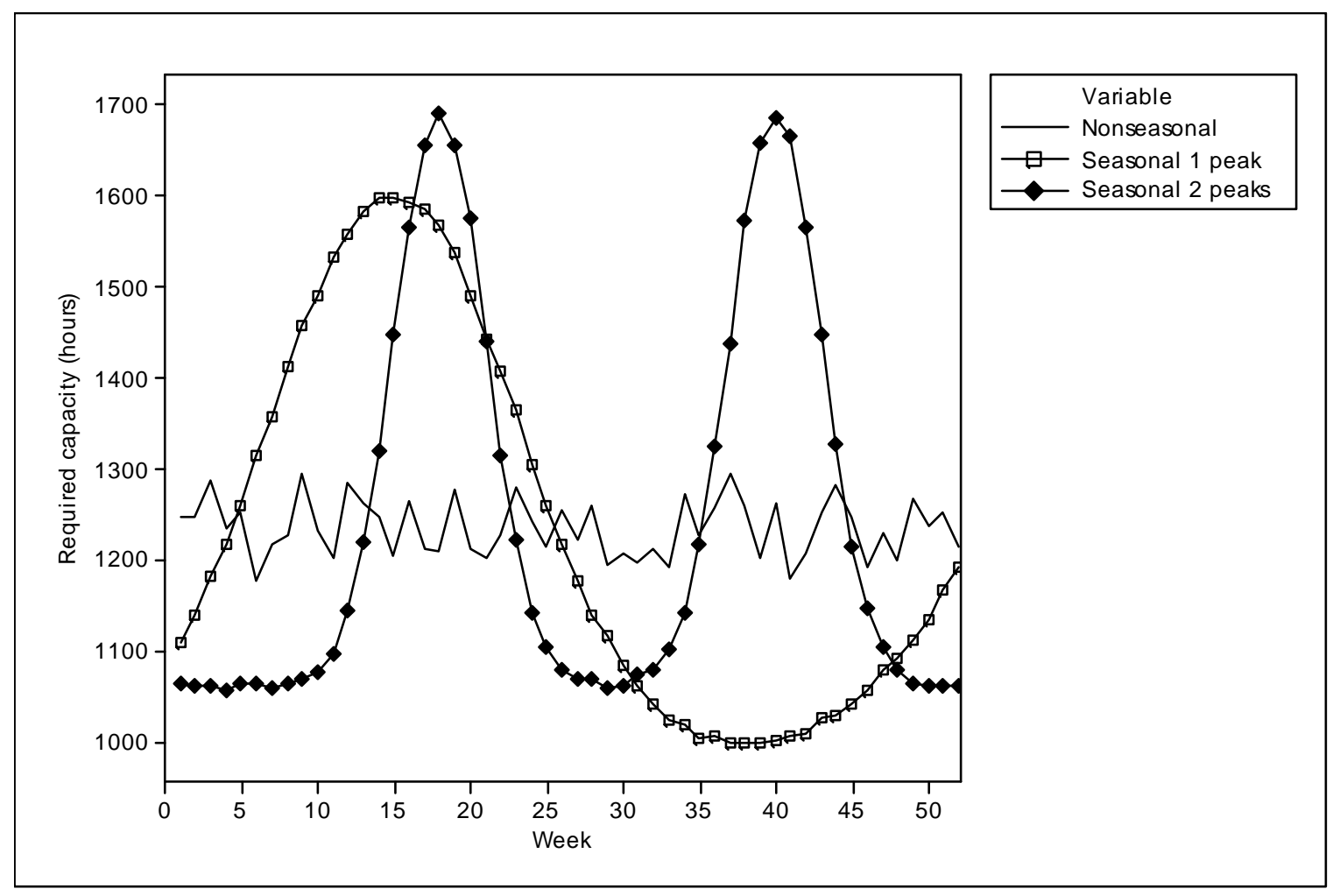

Figure 4: Required capacity patterns 
Figure 4: Required capacity patterns 
FIGURE 5

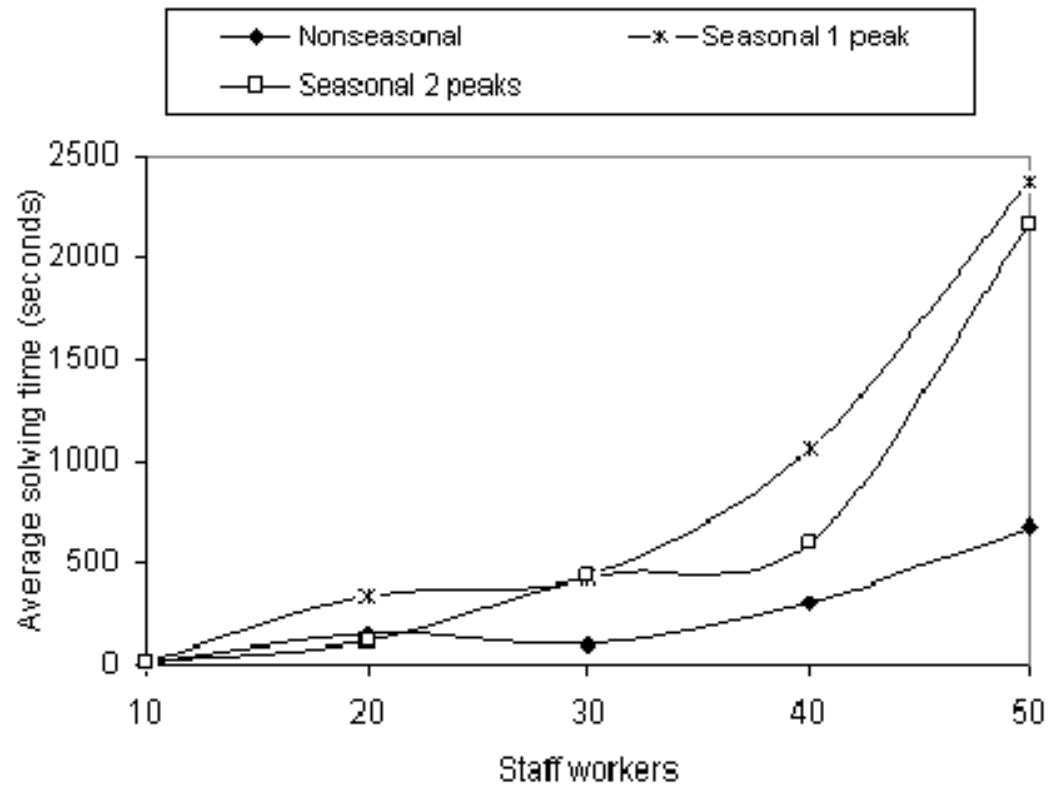

Figure 5: Influence of pattern of required capacity on computing times. 
Figure 5: Influence of pattern of required capacity on computing times. 


\section{TABLE 1}

Table 1: Size of the models.

\begin{tabular}{|c|c|c|c|c|}
\hline $\mathbf{M}$ & $|W|$ & $\begin{array}{c}\text { Number of } \\
\text { binary variables }\end{array}$ & $\begin{array}{c}\text { Number of } \\
\text { real variables }\end{array}$ & $\begin{array}{c}\text { Number of } \\
\text { constraint } \\
\text { s }\end{array}$ \\
\hline \multirow{5}{*}{ M1 } & 10 & 10,603 & 56 & 829 \\
\hline & 20 & 21,183 & 56 & 1,499 \\
\hline & 30 & 31,763 & 56 & 2,169 \\
\hline & 40 & 42,343 & 56 & 2,839 \\
\hline & 50 & 52,923 & 56 & 3,509 \\
\hline \multirow{5}{*}{ M2 } & 10 & 9,615 & 728 & 18,576 \\
\hline & 20 & 19,005 & 1,238 & 36,276 \\
\hline & 30 & 28,395 & 1,748 & 53,976 \\
\hline & 40 & 37,785 & 2,258 & 71,676 \\
\hline & 50 & 47,175 & 2,768 & 89,376 \\
\hline
\end{tabular}


Table 1: Size of the models. 


\section{TABLE 2}

Table 2: Computing times (in seconds).

\begin{tabular}{|c|c|c|c|c|c|c|c|c|}
\hline Model & $|W|$ & $t_{\min }$ & $\bar{t}$ & $t_{\max }$ & $\% O p t$ & $\% 50 p t$ & $\%>5$ & $\%$ NoFeas \\
\hline \multirow{5}{*}{ M1 } & 10 & 7 & 12 & 61 & 100 & & & \\
\hline & 20 & 11 & 405 & 3,600 & 94 & 6 & & \\
\hline & 30 & 22 & 573 & 3,600 & 92 & 8 & & \\
\hline & 40 & 28 & 949 & 3,600 & 92 & 8 & & \\
\hline & 50 & 60 & 1,729 & 3,600 & 80 & \multicolumn{3}{|c|}{20} \\
\hline \multirow{5}{*}{ M2 } & 10 & & \multirow{5}{*}{3,600} & & & & 24.5 & 75.5 \\
\hline & 20 & & & & & & 4.5 & 95.5 \\
\hline & 30 & & & & & & 6.7 & 93.3 \\
\hline & 40 & & & & & & 20 & 80 \\
\hline & 50 & & & & & & 16 & 84 \\
\hline
\end{tabular}


Table 2: Computing times (in seconds). 


\section{TABLE 3}

Table 3: Maximum relative capacity shortages and average relative capacity shortages (model M1).

\begin{tabular}{|c|c|c|c|c|c|c|}
\hline \multicolumn{4}{|c|}{$\begin{array}{c}\text { Maximum relative capacity } \\
\text { shortage }(D)\end{array}$} & \multicolumn{3}{|c|}{$\begin{array}{l}\text { Average relative capacity } \\
\qquad \begin{array}{c}\frac{1}{T} \cdot \sum_{t=1}^{T} \frac{d_{t}^{-}}{e_{t}} \\
\text { shortage }\end{array}\end{array}$} \\
\hline$|W|$ & Nonseasonal & $\begin{array}{c}\text { Seasonal } \\
1 \text { peak }\end{array}$ & $\begin{array}{l}\text { Seasonal } \\
2 \text { peaks }\end{array}$ & Nonseasonal & $\begin{array}{c}\text { Seasonal } \\
1 \text { peak }\end{array}$ & $\begin{array}{c}\text { Seasonal } \\
2 \text { peaks }\end{array}$ \\
\hline 10 & 0.35 & 0.28 & 0.25 & 0.055 & 0.046 & 0.04 \\
\hline 20 & 0.26 & 0.17 & 0.15 & 0.026 & 0.025 & 0.02 \\
\hline 30 & 0.35 & 0.24 & 0.25 & 0.032 & 0.026 & 0.022 \\
\hline 40 & 0.28 & 0.2 & 0.16 & 0.023 & 0.02 & 0.018 \\
\hline 50 & 0.22 & 0.18 & 0.14 & 0.02 & 0.02 & 0.022 \\
\hline
\end{tabular}


Table 3: Maximum relative capacity shortages and average relative capacity shortages (model M1). 